\title{
A novel surgical concept of transforaminal full-endoscopic lumbar undercutting laminectomy (TE-LUL) for central canal stenosis of the lumbar spine with local anesthesia: A case report and literature review
}

\author{
Koichi Sairyo, Kazuta Yamashita, Hiroaki Manabe, Yoshihiro Ishihama, Kosuke Sugiura, Fumitake Tezuka, \\ Yoichiro Takata, Toshinori Sakai, Yasuyuki Omichi, Nobutoshi Takamatsu, Ayaka Hashimoto, and Toru Maeda
}

Department of Orthopedics, Tokushima University, Tokushima, Japan

\begin{abstract}
Full-endoscopic spinal surgery was first developed for the lumbar herniated nucleus pulposus. Mainly, there are two types in the full-endoscopic lumbar surgery : i.e., transforaminal (TF) and interlaminar approach. The surgery can be done under the local anesthesia for the TF approach; therefore, we need to further develop the TF approach to variety of the spinal disorders. Recently, the TF full-endoscopic surgery has been applied for the spinal canal stenosis. First, transforaminal full-endoscopic lumbar foraminoplasty for the foraminal stenosis ; then, transforaminal lumbar lateral recess decompression for the lateral recess stenosis has been developed. Finally, we have developed the surgical technique to decompress the central stenosis via TF approach under the local anesthesia. Prior to initiate the clinical case, we have attempted the lumbar undercutting laminectomy using a fresh cadaveric spine. After we technically confirmed that the transforaminal full-endoscopic lumbar undercutting laminectomy (TE-LUL) is possible, we applied the technique to the patient whose lung capacity did not allow general anesthesia. The 72 years old female patient with central canal stenosis could be improved her left leg pain and muscle weakness after TE-LUL under the local anesthesia. In this paper, we introduce the surgical technique of the TE-LUL and discuss of the efficacy of the TE-LUL. J. Med. Invest. 66:224-229, August, 2019
\end{abstract}

Keywords : transforaminal full-endoscopic lumbar decompression, central stenosis, undercutting laminectomy

\section{INTRODUCTION}

Full-endoscopic lumbar spine surgery was initially developed using transforaminal (TF) approach. Several frontiers attempted to use an endoscope with a cannula $(1,2,3,4)$ using Hijikata's percutaneous discectomy technique (5). Finally, Dr. Yeung successfully established the current single-portal endoscopic discectomy $(3,4,6)$. The Full-endoscopic surgery requires only $8 \mathrm{~mm}$ skin incision, minimum damage to the back muscles and being able to conduct under local anesthesia $(7,8)$. Thus, it is understood the least invasive disc surgery, so far.

There is another way in the full-endoscopic surgery: i.e., interlaminar (IL) approach (9,10). It is called Interlaminar full-endoscopic lumbar discectomy (IELD). This is further minimally invasive surgery of microendoscopic discectomy (11). Recently, the IL technique has been applied for the decompression of the lumbar spinal canal stenosis $(12,13)$. Although we use same endoscopic system both for TF and IL approach, there is the biggest difference between them. The IL approach basically necessitates general anesthesia, on the other hand, the TF approach can be done under the local anesthesia with aware and awake condition.

The TF approach has been also applied for the decompression of the lumbar spinal canal stenosis. Once again, the TF approach is possible with the local anesthesia ; thus, it should be the great benefit especially for the elderly cases with poor general condition. First, the foraminal stenosis was decompressed with TF

Received for publication June 3, 2019; accepted July 4, 2019.

Address correspondence and reprint requests to Koichi Sairyo, MD, PhD, Professor and Chairman Department of Orthopedics, Tokushima University Graduate School, 3-18-15 Kuramoto, Tokushima 770 8503, Japan and Fax: +81-88-633-0178. full-endoscopic system $(14,15)$. Now, it is called Transforaminal endoscopic lumbar foraminotomy (TELF). Second, very recently, lateral recess stenosis can be decompressed with $\mathrm{TF}$ approach $(16,17,18)$.

There is one more type of the lumbar spinal canal stenosis : i.e., central stenosis. We have developed the TF full-endoscopic decompression technique for the disorder. First, we established the endoscopic technique with a fresh cadaver; then, we applied the surgery to one female case whose general condition was really poor. We name the technique as transforaminal full-endoscopic lumbar undercutting laminectomy (TE-LUL). In this paper, we describe the technique and introduce the first case. Finally, we discussed some issues on this procedure.

\section{A SCHEMATIC PRESENTATION OF THE SURGICAL TECHNIQUE}

Figure 1 demonstrates the surgical procedure of the TE-LUL. The $8 \mathrm{~mm}$ cannula is first installed on the superior articular process (SAP). Total removal of the SAP is performed at the step 1 ; then, articular surface of the inferior articular process (IAP) is exposed. Next, undercutting laminectomy is conducted with ventral half resection of the IAP as the step II. After that, the dorsal surface of ligamentum flavum is seen. Almost of the ligamentum flavum at the area is removed as the step III, and decompression of the central stenosis is completed. As shown in the final panel on Figure 1, the compression of the dural tube is enlarged after the TE-LUL surgery. 


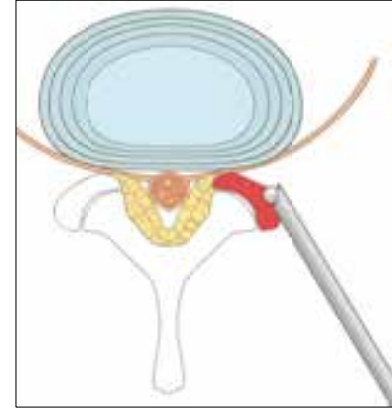

Step I :

Total removal of SAP

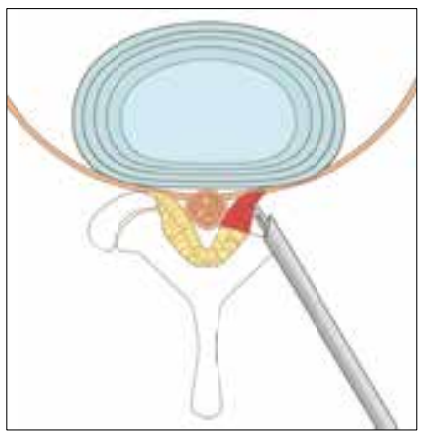

Step III :

Partial removal of $\mathrm{LF}$

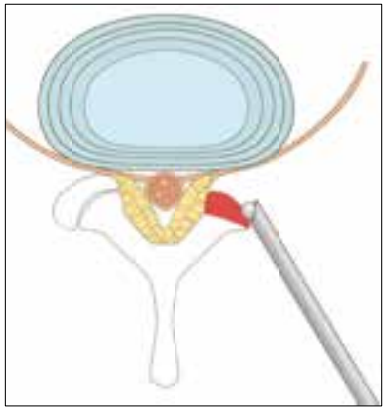

Step II :

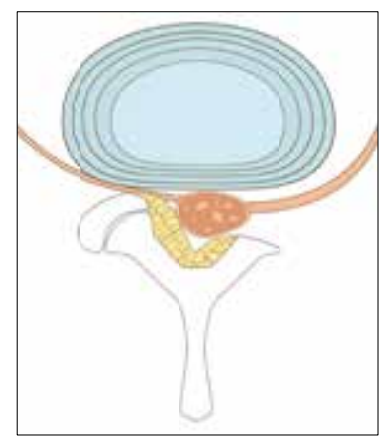

Step IV :

Final view of TF-LUL
Partial removal of IAP

Figure 1. Surgical procedure of the TE-LUL.

The $8 \mathrm{~mm}$ cannula is first installed on the superior articular process (SAP). Total removal of the SAP (color in red) is performed at the step 1. Undercutting laminectomy (color in red) is conducted with ventral half resection of the IAP as the step II. The ligamentum flavum at the area (color in red) is partially removed as the step III. As shown in the final panel on, the compression of the dural tube is enlarged after the TE-LUL surgery.

\section{A CASE REPORT}

A 72 years old female visited us with complaining of both legs pain and left leg weakness. Standing upright posture caused severe legs pain; thus, she was using whirl chair with lumbar flexed position to move. Any of conservative treatment was not effective to reduce her symptom for 2 months; then, she was referred to us for the purpose of surgical intervention.

On her first visit, we examined motor manual testing and found muscle weakness on the left side as follows : quadriceps $(4 / 5)$, tibialis anterior (2/5), and extensor halluscis longus (2/5), whereas the right side showed all normal. Figure 2,3, and 4 demonstrates MRIs before and after the surgery. Most severe stenosis is found at L3/4, and at the other levels stenosis was very moderate. Thus, we diagnosed that her symptoms were due to cauda equina syndrome due to the central canal stenosis at L3/4. We planned posterior decompression with laminectomy and flavectomy under the general anesthesia.

Before surgery, an anesthesiologist examined her general condition. The physician pointed that her lung capacity was so weak : i.e. one second volume being 0.71 (l), that she could not tolerate the general anesthesia. Thus, the anesthesiologist recommended her to avoid the general anesthesia and to undergo any kinds of surgery that can be done under the local anesthesia. Under the guidance of the anesthesiologist, we decided to conduct the TE-LUL for L3/4 bilaterally.

First, we performed to the left side, since muscle weakness

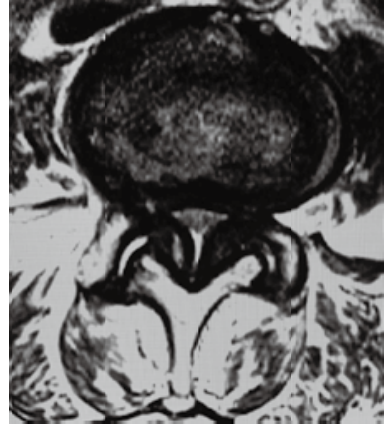

Pre-OP

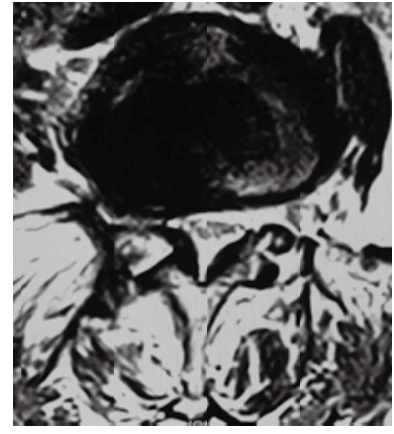

Post-OP
Figure 2. The comparative axial MRI before and after the surgery. Decompression is obtained with TE-LUL on the axial image.

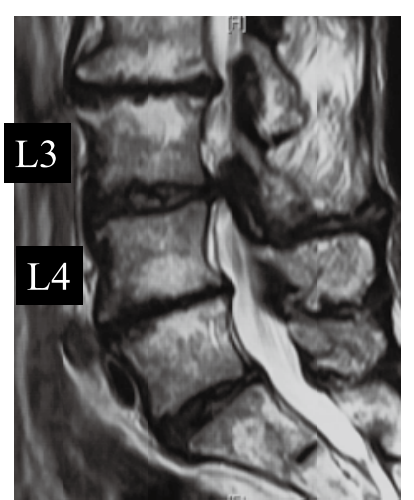

Pre-OP

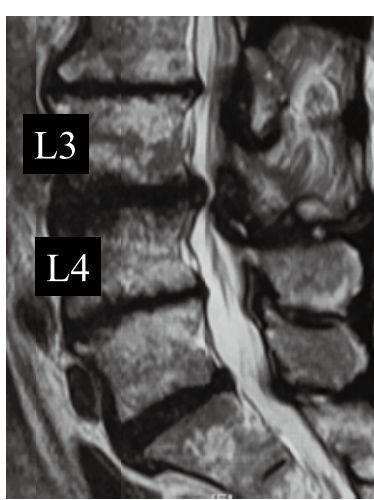

Post-OP
Figure 3. The comparative right para-sagittal MRI before and after the surgery.

Although slight compression of the dural tube is still existed, complete compression before the surgery was recovered after the surgery.

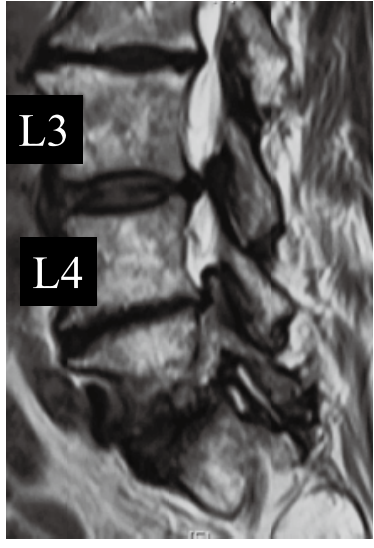

Pre-OP

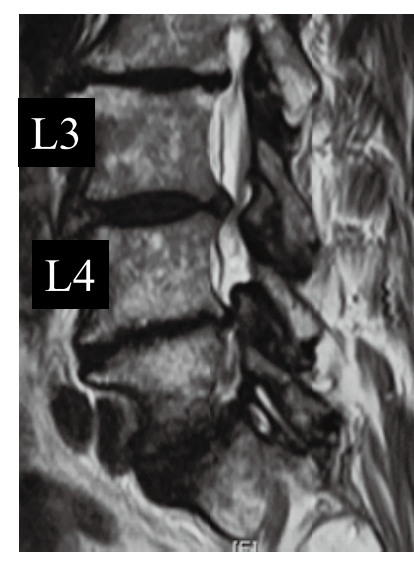

Post-OP
Figure 4. The comparative left para-sagittal MRI before and after the surgery.

Although slight compression of the dural tube is still existed, complete compression before the surgery was recovered after the surgery.

was present at the left. Local anesthesia was conducted to skin, subcutaneous tissue, muscle, capsule, facet joint and the surface of the disc with $15 \mathrm{ml}$ of $1 \%$ lidocaine. Following skin incision in 8 $\mathrm{mm}$, a cannula was installed just on the SAP joint. TE-LUL was conducted with the technique as shown in Figure 1. After the 
confirmation of the decompression of L4 traversing nerve root and dura matter, TE-LUL for the left side was over. The right side was also decompressed with TE-LUL maneuver. During the surgery, we did not experience any complications. One hour after the surgery, she stood and walked with a walker. She noticed no leg pain any more, and rehabilitation started for her muscle weakness. One week later, the weakness partially improved to quadriceps (5/5), tibialis anterior (4/5), and extensor halluscis longus (3/5). She returned to her job as a president of a local company two weeks after the surgery, since low back and leg pain disappeared. Two months later, strength of the tibialis anterior and extensor halluscis longus completely became normal.

Figure 2,3, and 4 demonstrates the comparative MRI before and after the surgery. Decompression is obtained with TE-LUL on the axial image as shown in Figure 2. On the right (Figure 3) and the left (Figure 4) sagittal images, slight compression of the dural tube is still existed. However, complete compression before the surgery was recovered after the surgery.

CT scans before and after the surgery were exhibited on Figure 5 (3-dimensional CT), Figure 6 (sagittal CT) and Figure 7 (axial CT). On posterior view of the 3-dimensional CT, it is very difficult to understand the decompression area, since during the surgery posterior construct was mostly preserved (Figure 5). On the sagittal view, it is obvious that entire SAP and partial IAP is resected at L3-4 level bilaterally (Figure 6). Even though entire SAP is removed, facet contact is about $50 \%$ remained. On axial scans, one can easily understand that entire SAP and partial IAP is resected (Figure 7).

\section{DISCUSSION}

In the literature, there are many reports to conduct full-endoscopic decompression of the central stenosis under the general anesthesia via interlaminar approach $(12,13)$. In this paper, we have first introduced the transforaminal full-endoscopic surgical technique to decompress the central canal stenosis under the local anesthesia as shown in Figure 1. We have named this technique transforaminal full-endoscopic lumbar undercutting laminectomy (TE-LUL).

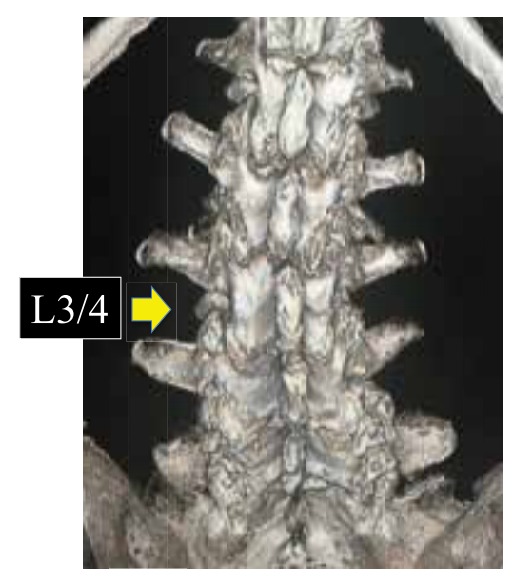

Pre-OP

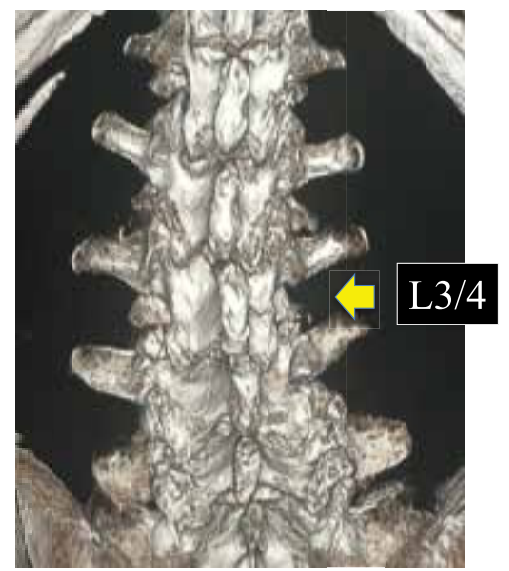

Post-OP

Figure 5. Posterior view of the three dimensional CTs before and after the surgery. It is very difficult to understand the decompression area, since during the surgery posterior construct was mostly preserved

Right

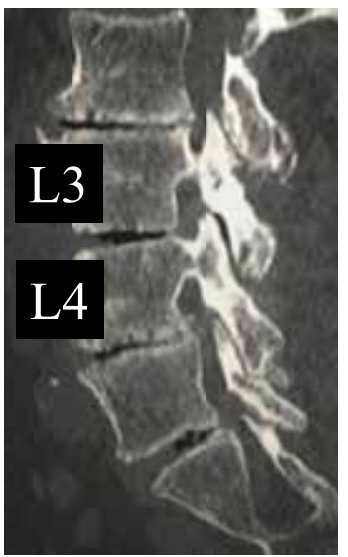

Pre-OP

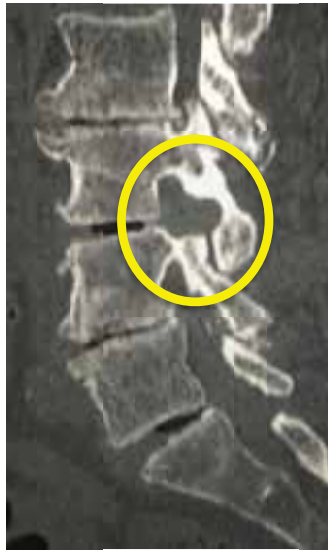

Post-OP

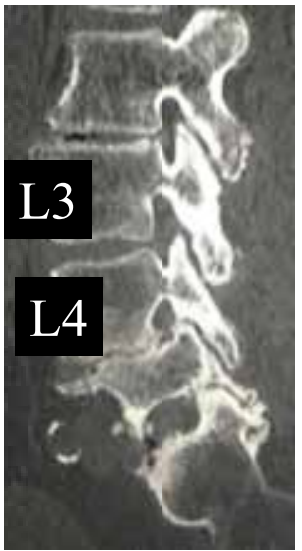

Pre-OP
Left

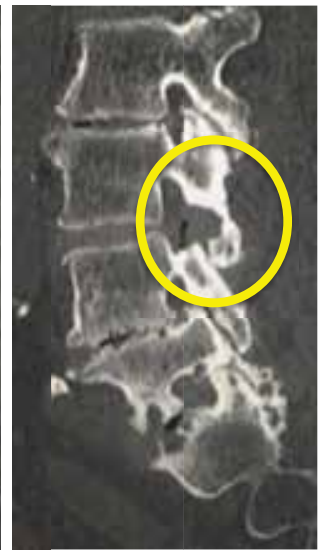

Post-OP

Figure 6. The sagittal reconstructive CTs before and after the surgery.

It is obvious that entire SAP and partial IAP is resected at L3-4 level bilaterally. Even though entire SAP is removed, facet contact is about $50 \%$ remained. 


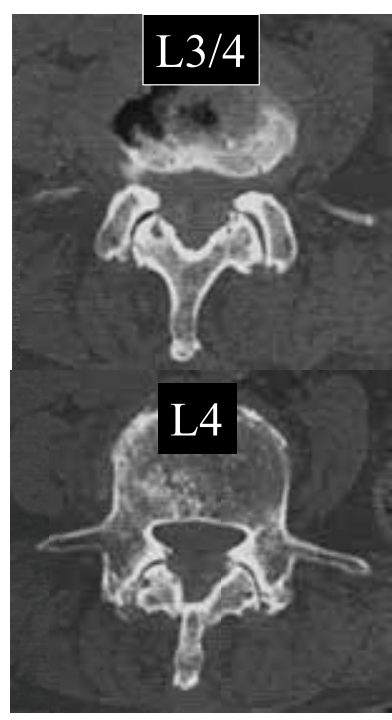

Pre-OP

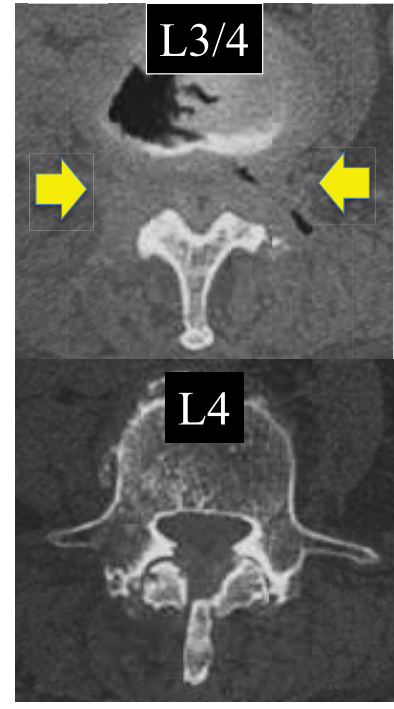

Post-OP
Figure 7. The axial reconstructive CTs before and after the surgery. On axial scans, one can easily understand that entire SAP and partial IAP is resected.

The great benefit of the TE-LUL surgery should be what one can perform this procedure under the local anesthesia with just $8 \mathrm{~mm}$ skin incision. The lumbar spinal canal stenosis is the disorder for the elderly patients. As comparing to the younger generation, elderly patients has poor tolerance for the general anesthesia. For example, they may frequently have disorders in cardiovascular, pulmonary, hepatic, renal, and/or cognitive function. Among them, pulmonary dysfunction would be extremely important like the current case.

Chung et al. (19) reviewed 404 elderly cases who underwent surgery under the general anesthesia and evaluated post-operative complications. They found that $26.4 \%$ of patients developed one or more postoperative complications, and the in-hospital mortality rate was $6.7 \%$. Respiratory complication was the most common postoperative complication (12.9\%). They also evaluated the effectiveness of the anesthesia method on the post-operative pulmonary complication and found that the regional anesthesia could reduce the complication as comparing to the general anesthesia. Rock and Rich (20) reviewed post-operative pulmonary complications (POPC) and concluded that POPC is a significant source of morbidity and mortality, and regarding the risk factors they stated that cigarette smoking, chronic respiratory disease, age greater than 70 years, and others. Thus, the elderly patients with pulmonary dysfunction like the current case has a great risk for the post-operative pulmonary complication.

Urwin et al. (21) reviewed 2162 cases from 15 trials who underwent hip surgery under regional or general anesthesia. They concluded that there are advantages for regional anaesthesia compared to general anaesthesia in terms of early mortality. Rasmussen et al. (22) reviewed 438 elderly patients who had major non-cardiac surgery. The 217 cases surgeries under the general anesthesia, and the remaining 211 underwent surgeries under the regional anesthesia. They found that the mortality after the general anesthesia was significantly higher. Chou et al. (23) reviewed 2,407 registered cases of femoral fracture in the aged (over 65 y.o.). They found 42 mortal patients, and found the patients in mortality group had a significantly higher rate of pneumonia, respiratory failure, sepsis, and pulmonary embolism than those in the survival groups. Regarding the mortality after the surgery for elderly patients, especially for poor pulmo- nary condition, spine surgeons need to develop the spinal decompression surgery that can be done under the local anesthesia.

Thanks to many frontiers on the endoscopic spine surgeries, recently, single portal full-endoscopic spine surgery has been developed and established $(1,2,3,4,6)$. At first, the full-endoscopic surgery was limited only for the disc surgery $(3,4,6,7,8,9,10)$; however, the technique has been applied for the lumbar spinal canal stenosis (LSS). There are two maneuver in the full-endoscopic surgery : i.e. interlaminar (IL) and tansforaminal (TF). Indeed, the decompression for the LSS with IL full-endoscopic technique would be minimally invasive $(12,13)$. Although the trial of IL surgery under the local anesthesia (24) has been reported, majority of the IL surgery requires general anesthesia $(12,13)$.

The TF full-endoscopic surgery is possible to conduct under the local anesthesia ; thus, one needs to expand the surgical indication from the disc surgery to the LSS decompression surgery. First, the foraminal stenosis has be overcome with the TF technique $(14,15,25)$. Yeung et al. (25) reported the long-term clinical results and more than $80 \%$ would be excellent and good. Next, TF full-endoscopic lateral recess decompression (TE-LRD) was started $(16,17,18,26,27,28)$. Ahn et al. (26) followed 45 patients who had the TE-LRD for years and reported satisfactory clinical results more than $80 \%$. Thus, for the foraminal and lateral recess stenosis, technique and skill of the TF full-endoscopic surgery would be now established.

There is one more type of the LSS : i.e. central canal stenosis. There has been no report in the literature on the TF full-endoscopic technique of the decompression of the central canal stenosis. This time, we have developed such technique and named transforaminal full-endoscopic lumbar undercutting laminectomy (TE-LUL). Finally, all three types of the LSS is possible to be decompressed with transforaminal full-endoscopic surgery under the local anesthesia.

\section{LIMITATIONS}

There are possible limitations in the TE-LUL surgery.

1) indication of the surgery

By the TE-LUL surgery, it is very difficult to remove entire ligamentum flavum (LF). As shown in Figure 3 and 4, partial LF still exists after the surgery. Thus, the first indication should be the traditional interlaminar laminectomy with the general anestheisa. This time, the case was not combined with the spondylolisthesis. Thus, the surgical outcome for the LSS patients with spondylolisthesis is still unclear.

2) post-operative complication

The possible concern after the total removal of SAP would be instability following TE-LUL. However, as shown in Figure 6 , after the total SAP resection, about $50 \%$ of the facet contact could be preserved. Moreover, the cases that is in a good indication should be not active and poor general condition. Post-surgical instability may not be occurred. The biggest concern would be fatigue fracture of the thinner SAP after the surgery. We warned this patient not to perform the repetitive extension exercise.

\section{CONCLUSION}

In this paper, we have proposed a novel surgical concept of transforaminal full-endoscopic lumbar undercutting laminectomy (TE-LUL) for central canal stenosis of the lumbar spine under local anesthesia, and reported the clinical results in the initial case. There are three types of the lumbar spinal canal stenosis. TELF for the foraminal stenosis, TE-LRD for the lateral 
recess stenosis, and TE-LUL for the central stenosis. Now, for the standard three type of the stenosis, transforaminal full-endoscopic decompression under the local anesthesia is finally developed.

\section{ACKNOWLEDGMENT}

The authors wish to thank to the following people and companies for their support to develop this new endoscopic technique.

Daido Nakahara, Yasuo Araki, Akihito Kobayashi, Takeshi Fujiwara, Eitaro Tanaka, Shigeki Tenma, Yozo Takehisa, Kazue Kobayashi, Sei-Ichi Mima, Keiko Shimada, Takahiko Makino, Shigeru Ueda, Yoshiyuki Kato, Fujio Ito, Michio Ueda, Yuiji Ogawa, Masao Marumoto, Nobuaki Mima, Tomoni Mima, Tatsuo Kosugi, Ken Noguchi, Sumiko Suzuki, Hachioji Sports Clinic, and Nihon Flush Co., Ltd.

\section{REFERENCES}

1. Kambin P, Schaffer JL: Percutaneous lumbar discectomy. Review of 100 patients and current practice. Clin Orthop Relat Res 238: 24-34, 1989.

2. Schreiber A, Leu H:Percutaneous nucleotomy : technique with discoscopy. Orthopedics. 1991 Apr ; 14(4) : 439-44.

3. Yeung AT : The evolution of percutaneous spinal endoscopy and discectomy : state of the art. Mt Sinai J Med $67: 327$ 32,2000

4. Yeung AT, Tsou PM : Posterolateral endoscopic excision for lumbar disc herniation : Surgical technique, outcome, and complications in 307 consecutive cases. Spine 27 : 722 -31, 2002.

5. Hijikata S : Percutaneous nucleotomy. A new concept technique and 12 years' experience. Clin Orthop Relat Res 238: 9-23, 1989 .

6. Yeung AT, Yeung CA : Minimally invasive techniques for the management of lumbar disc herniation. Orthop Clin North Am 38(3) : 363-72, 2007

7. Sairyo K, Nagamachi A. State-of-the-art management of low back pain in athletes : Instructional lecture. J Orthop Sci. 2016 May ; 21(3) : 263-72.

8. Choi G, Lee SH, Lokhande P, Kong BJ, Shim CS, Jung B, Kim JS. Percutaneous endoscopic approach for highly migrated intracanal disc herniations by foraminoplastic technique using rigid working channel endoscope. Spine (Phila Pa 1976). 2008 Jul 1 ; 33(15) : E508-15.

9. Ruetten S, Komp M, Merk H, et al. Full-endoscopic interlaminar and transforaminal lumbar discectomy versus conventional microsurgical technique : a prospective, randomized, controlled study. Spine (Phila Pa 1976) 2008; 33 : 931 -9.

10. Birkenmaier C, Komp M, Leu HF, et al. The current state of endoscopic disc surgery : review of controlled studies comparing full-endoscopic procedures for disc herniations to standard procedures. Pain Physician 2013 ; 16 : 335 -44.

11. Foley KT, Smith MM : Microendoscopic discectomy. Tech Neurosurg 3: 301 - 307, 1997

12. Komp M, Hahn P, Merk H, Godolias G, Ruetten S. Bilateral operation of lumbar degenerative central spinal stenosis in full-endoscopic interlaminar technique with unilateral approach : prospective 2-year results of 74 patients. J Spinal Disord Tech. $2011 \mathrm{Jul}$; 24(5) : 281-7.

13. Lee CW, Yoon KJ, Jun JH. Percutaneous Endoscopic Laminotomy with Flavectomy by Uniportal, Unilateral Approach for the Lumbar Canal or Lateral Recess Stenosis. World Neurosurg. 2018 May ; 113 : e129-e137.
14. Yamashita K, Higashino K, Sakai T, Takata Y, Hayashi F, Tezuka F, Morimoto M, Chikawa T, Nagamachi A, Sairyo K. Percutaneous full endoscopic lumbar foraminoplasty for adjacent level foraminalstenosis following vertebral intersegmental fusion in an awake and aware patient under local anesthesia : A case report. J Med Invest. 2017 ; 64(3.4) : 291-295.

15. Yeung A, Gore S. Endoscopic foraminal decompression for failed back surgery syndrome under local anesthesia. Int J Spine Surg. 2014 Dec 1; 8. doi : 10.14444/1022. eCollection 2014.

16. Sairyo, K., K. Higashino, K. Yamashita, F. Hayashi, K. Wada, T. Sakai, Y. Takata, F. Tezuka, M. Morimoto, T. Terai, T. Chikawa, H. Yonezu, A. Nagamachi and Y. Fukui (2017). "A new concept of transforaminal ventral facetectomy including simultaneous decompression of foraminal and lateral recess stenosis : Technical considerations in a fresh cadaver model and a literature review.” J Med Invest 64(1.2) : 1-6.

17. Lewandrowski, K. U. (2018). "Endoscopic Transforaminal and Lateral Recess Decompression After Previous Spinal Surgery." Int J Spine Surg 12(2) : 98-111.

18. Nakajima D, Yamashita K, Tezuka F, Manabe H, Ishihama Y, Sugiura K, Takata Y, Sakai T, Maeda T, and Sairyo K. Successful percutaneous endoscopic decompression surgery under the local anesthesia for L5 radiculopathy caused by L5-S1 foraminal stenosis and L4-5 lateral recess stenosis : A case report. J Med Invest, in press.

19. Chung JY, Chang WY, Lin TW, Lu JR, Yang MW, Lin CC, Chang CJ, Chou AH. An analysis of surgical outcomes in patients aged 80 years and older. Acta Anaesthesiol Taiwan. 2014 Dec ; 52(4) : 153-8.

20. Rock P, Rich PB. Postoperative pulmonary complications. Curr Opin Anaesthesiol. 2003 Apr ; 16(2) : 123-31.

21. Urwin SC, Parker MJ, Griffiths R. General versus regional anaesthesia for hip fracture surgery : a meta-analysis of randomized trials. Br J Anaesth. 2000 Apr ; 84(4) : 450-5.

22. Rasmussen LS, Johnson T, Kuipers HM, Kristensen D, Siersma VD, Vila P, Jolles J, Papaioannou A, Abildstrom H, Silverstein JH, Bonal JA, Raeder J, Nielsen IK, Korttila K, Munoz L, Dodds C, Hanning CD, Moller JT ; ISPOCD2(International Study of Postoperative Cognitive Dysfunction) Investigators. Does anaesthesia cause postoperative cognitive dysfunction? A randomised study of regional versus general anaesthesia in 438 elderly patients. Acta Anaesthesiol Scand. $2003 \mathrm{Mar}$; 47(3) : 260-6.

23. Chou SE, Rau CS, Tsai YC, Hsu SY, Hsieh HY, Hsieh CH. Risk Factors and Complications Contributing to Mortality in Elderly Patients with Fall-Induced Femoral Fracture : A Cross-sectional Analysis Based on Trauma Registry Data of 2,407 patients. Int J Surg. 2019 Apr 23. pii : S17439191(19)30093-7. doi : 10.1016/j.ijsu.2019.04.010. [Epub ahead of print]

24. Youn MS, Shin JK, Goh TS, Son SM, Lee JS. Endoscopic posterior decompression under local anesthesia for degenerative lumbar spinal stenosis. J Neurosurg Spine. 2018 Dec $1 ; 29(6): 661-666$.

25. Yeung A, Roberts A, Zhu L, Qi L, Zhang J, Lewandrowski KU. Treatment of Soft Tissue and Bony Spinal Stenosis by a Visualized Endoscopic Transforaminal Technique Under Local Anesthesia. Neurospine. 2019 Mar ; 16(1) : 52-62. doi : 10.14245/ns.1938038.019. Epub 2019 Mar 31.

26. Ahn Y, Keum HJ, Lee SG, Lee SW. Transforaminal Endoscopic Decompression for Lumbar Lateral Recess Stenosis : An Advanced Surgical Technique and Clinical Outcomes. World Neurosurg. 2019 Feb 11. pii : S1878- 
8750(19)30320-1. doi : 10.1016/j.wneu.2019.01.209. [Epub ahead of print]

27. Yang J, Wu H, Kong Q, Wang Y, Peng Z, Zhang L, Yan Y, Guo C, Zhang D. Full Endoscopic Transforaminal Decompression Surgery for Symptomatic Lumbar Spinal Stenosis in Geriatric Patients. World Neurosurg. 2019 Mar 25. pii :
S1878-8750(19)30851-4. doi : 10.1016/j.wneu.2019.03.171. [Epub ahead of print]

28. Sairyo K, Chikawa T, Nagamachi A. State-of-the-art transforaminal percutaneous endoscopic lumbar surgery under local anesthesia: Discectomy, foraminoplasty, and ventral facetectomy. J Orthop Sci. 2018 Mar ; 23(2) : 229-236. 